\title{
Exosomes as therapeutic vehicles in liver diseases
}

\author{
Jingyi Ding, Ju Wang, Jiajia Chen^
}

State Key Laboratory for Diagnosis and Treatment of Infectious Diseases, National Clinical Research Center for Infectious Diseases, Collaborative Innovation Center for Diagnosis and Treatment of Infectious Diseases, The First Affiliated Hospital, Zhejiang University School of Medicine, Hangzhou, China

Contributions: (I) Conception and design: J Chen; (II) Administrative support: None; (III) Provision of study materials or patients: None; (IV) Collection and assembly of data: None; (V) Data analysis and interpretation: None; (VI) Manuscript writing: All authors; (VII) Final approval of manuscript: All authors.

Correspondence to: Jiajia Chen. The First Affiliated Hospital, Zhejiang University School of Medicine, No. 79 Qingchun Road, Hangzhou 310003 , China. Email: jiajiatale0@zju.edu.cn.

\begin{abstract}
The diagnosis and treatment of various liver diseases have progressed greatly over the years, but clinical outcomes are still not satisfying. New research on the mechanisms and application thereof may effectuate positive changes. Exosomes are membrane-derived nanovesicles ranging in size from 40 to $160 \mathrm{~nm}$ and are released by a diversity of cells. They contain a variety of cargo, including lipids, proteins, coding RNAs, and noncoding RNAs. Recent studies have recognized exosomes as intercellular communication agents, which play important roles in physiological or biological processes in acute or chronic liver disorders by horizontal transferring of genetic bioinformation from donor cells to neighboring or distal target cells. In the hope that exosomes can potentially be used as vehicles for clinical intervention, this review aims to focus on the roles of exosomes and their cargo in the field of various liver disorders, including virus-related liver diseases, alcoholic liver diseases (ALD), nonalcoholic fatty liver diseases (NAFLD), and liver cancer. In addition, many studies have indicated that mesenchymal stem cell (MSC)-derived exosomes or engineered MSC-derived exosomes can also exert hepatoprotection, antioxidation, or enhance drug sensitivity on corresponding liver diseases with the advantage of low immunogenicity and high biocompatibility. Overall, exosomes are expected to serve as an important therapeutic tool for various liver diseases. However, there are still many problems that need to be resolved by further research and a greater body of evidence before exosomes are ready for clinical application.
\end{abstract}

Keywords: Exosomes; miRNA; mesenchymal stem cell (MSC); liver disease

Submitted Jul 23, 2020. Accepted for publication Feb 08, 2021.

doi: $10.21037 / \mathrm{atm}-20-5422$

View this article at: http://dx.doi.org/10.21037/atm-20-5422

\section{Introduction}

Exosomes were first described as a means to eliminate cellular waste by Pan et al. in 1985 (1). While studying the maturation process of erythrocytes, they found that exosomes served to remove unnecessary components from cells. The terms "exosome" and "extracellular vesicle (EV)" were once used interchangeably in many publications. However, it is now widely accepted that an exosome is a membrane vesicle structure ranging from 40 to $160 \mathrm{~nm}$ in size that originates from multivesicular bodies (MVBs) through endosomal pathways, which consist of endosome formation and exocytosis (2). The formation of MVBs starts from the early sorting of endosomes (ESEs) which are generated from the invagination of plasma membrane and the endocytosis of biomolecules (3). After periods of maturation, the late endosomal membrane buds inward

^ ORCID: 0000-0001-9701-8677. 
to form the intraluminal vesicles (ILVs), which leads to the formation of MVBs (4). Some of MBVs can be fused with plasma membrane, and subsequently release ILVs into the extracellular space, and these can be defined as exosomes (2,5-7). Other MBVs can be fused with lysosomes or autophagosomes for self-degradation (3) (Figure 1, A). Recent research $(8,9)$ has recognized that exosomes serve as intercellular communication agents which contain various biomolecules, such as lipids, proteins, amino acids, coding RNAs, and noncoding RNAs. In this capacity, exosomes play critical roles in physiological or biological processes in acute or chronic liver disorders by the horizontal transfer of genetic bioinformation from donor cells to neighboring or distal target cells. Other studies (4) have shown that exosomes can be secreted by the majority of cell types. Depending on the cell types released, the quantity, contents, and biological characteristics of exosomes can promote or inhibit the physiological or pathological progression of cells. For instance, normal hepatocytes were found to release a small number of exosomes to regulate liver repair and regeneration (10). Meanwhile, stressed hepatocytes were found to increase exosome release and the expression of cellular mRNA, which modulates the transcriptional process of adjacent hepatocytes and hepatic stellate cells (HSCs) (11). Exosomes released by nonparenchymal cells, such as sinusoidal endothelial cells, Kupffer cells, and HSCs, also participate in the regulation of liver function physiologically or pathologically. Moreover, liver tumor cells exert great influence on regulating the growth, angiogenesis, proliferation, and metastasis of neoplasm by releasing exosomes (12). Such exosome-modulated responses can be disease promoting or suppressing (3).

Accumulating evidence $(13,14)$ has clarified the functions of exosomes in the hepatic pathological state: due to their low immunogenicity and good biocompatibility, exosomes may transfer biomolecules to the target cells without RNA degradation and loss of biological information. Thus, there has been increasing emphasis on the use of exosomes to control the progression of liver diseases. There are three main ways in which exosomes can be manipulated to facilitate liver regeneration, regulate inflammation and fibrosis, or inhibit tumor growth and metastasis: (I) direct regulation of the release of exosomes from particular cell types $(15,16)$; (II) interference with exosome cargo (17); and (III) delivery of drugs into exosomes (18). Indeed, the study of exosomes has become a very active field. Ongoing experiments may discover more about exosome functions, which may in turn help to enhance the diagnosis and treatment of a variety of diseases (3).

\section{Hepatitis B}

Hepatitis B virus (HBV) infection has become a major global health problem (19). According to the World Health Organization (WHO) Global Hepatitis Report 2017, 257 million people were estimated to be chronically infected with HBV worldwide. People who are chronically infected with HBV may develop cirrhosis or hepatocellular carcinoma (HCC), a potentially fatal disease. Hence, it is crucial to understand the pathogenesis of $\mathrm{HBV}$ in order to better intervene in liver diseases. After HBV reaches the liver and infects the hepatocytes through the bloodstream, its envelope fuses with the hepatocyte membrane, and the HBV genome, a partially doublestranded, relaxed circular DNA (rcDNA), is transported to the nucleus of host hepatocytes and converted into covalently closed circular DNA (cccDNA) which serves as the template to synthesize the pregenomic mRNA after multiple steps (20-22). HBV DNA is then created by reverse transcription and may be integrated into the chromosome. Based on these characteristics, exploring the roles of exosomes in HBV replication, transmission, and immune responses is particularly important. HBV X protein $(\mathrm{HBx})$, a multifunctional viral regulator, figures prominently in HBV duplication and viral carcinogenesis by interacting with host factors (19). It can surpass the host exosomal biogenesis mechanism by enhancing the activity of neutral sphingomyelinase 2 and interacting with foreign biomarkers such as neutral sphingomyelinase 2, CD9, and CD81 (23). Moreover, a growing body of evidence suggests that exosomes that contain the HBV genome can be released from infected hepatocytes to adjacent normal cells, leading to the spread of virus (24,25). Yang et al. (26) highlighted that carboxyfluorescein diacetate succinimidyl ester (CFSE)-labeled HLCZ01 cells incubated with HBVpositive exosomes labeled with 4-chlorobenzenesulfnate salt (DiD) were shown to be positive for hepatitis B surface antigen (HBsAg) and core antigen ( $\mathrm{HBcAg}$ ) after 2 days of exposure. We can thus infer that the spread of the virus can be blocked by regulated exosomes. Furthermore, Wang et al. (27) confirmed that the exosome-mediated clustered regularly interspaced short palindromic repeats (CRISPR)CRISPR-associated protein 9 (Cas9) system could cut the intercellular transmission function of the HBV genome (Figure 1, A). Additionally, exosomes released after virus infection can also modulate immune responses to control 


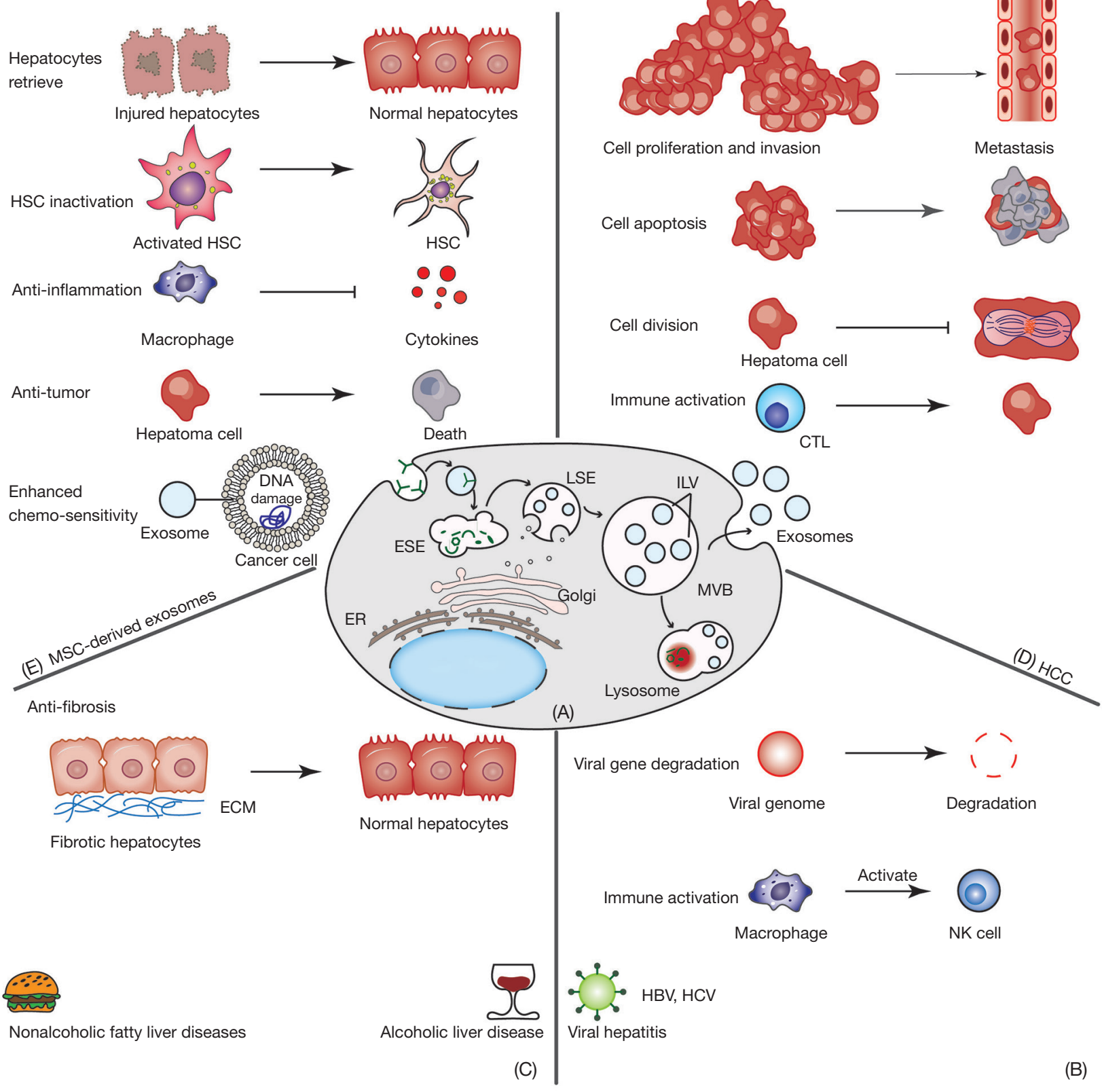

Figure 1 Role of exosomes in liver diseases. (A) The biogenesis of exosomes. Exosomes are generated from the formation of ESE, LSE, and MVBs in sequence. In cytoplasm, ILVs are either degraded by lysosomes or released to the extracellular environment as exosomes. (B) Exosomes exert antiviral effects during viral hepatitis. Exosomes could induce viral genome degradation and activate immune cells. (C) Exosomes have functions in antifibrosis in alcoholic liver disease and nonalcoholic fatty liver diseases. (D) Role of exosomes in HCC. Exosomes participate in the inhibition of tumor growth, metastasis, cell division, and the activation of CTL. (E) Multiple roles of MSCderived exosomes. MSC-derived exosomes involved in injured hepatocyte repair, HSC inactivation, anti-inflammation, and antitumor and chemosensitivity promotion. ESE, early sorting endosome; LSE, late sorting endosome; MVB, multivesicular bodies; ILV, intraluminal vesicle; HCC, hepatocellular carcinoma; MSC, mesenchymal stem cell; HSC, hepatic stellate cell; ECM, extracellular matrix; NK cell, natural killer cell; CTL, cytotoxic T cell; ER, endoplasmic reticulum; Golgi, Golgi apparatus. 
the virus (28). Kouwaki et al. (29) discovered that exosomes containing viral genes derived from $\mathrm{HBV}$-infected hepatocytes in vitro could induce the expression of natural killer group 2, member D (NKG2D) ligand in macrophages through myeloid differentiation factor 88 (MyD88), TIR domain-containing adaptor molecule-1 (TICAM-1), and mitochondrial antiviral signaling (MAVS)-dependent pathways (Figure 1, B). The depletion of exosomes may significantly reduce the expression of NKG2D ligand, so as to postpone disease progression.

\section{Hepatitis C}

Hepatitis C virus (HCV), a small, enveloped RNA virus, can progress to chronic hepatitis, cirrhosis, or even HCC under long-term exposure (30). It is reported that approximately 170 million people suffering from this virus (31). Therefore, it is urgent to explore new treatments for HCV. Cosset et al. (32) reported that exosomes derived from HCVinfected hepatocytes (HCV-exo) had the ability to transmit pathogenic substances to human hepatoma HuH7.5.1 cells through resisting neutralizing antibodies. However, in an experiment of HCV-replicating Huh7.5 cells treated by the MVB inhibitor, U18666A, the quantity of the viral genomes released to the supernatant showed a significant decrease (31). $\mathrm{HCV}$-exo can also transfer viral nucleic acid from HCVinfected cells to adjacent immune cells, stimulate the expansion of myeloid-derived suppressor cells (MDSCs), subsequently promote the differentiation of $\mathrm{T}$ follicular regulatory cells (TFR), and inhibit the functions of $T$ follicular helper cells (TFH), all of which constitute a newly discovered mechanism of immune dysfunction in the process of chronic viral infection (33). Meanwhile, it was reported that the promotion of TFR differentiation and repression of TFH function were associated with the inhibition of miRNA (miR)-124 expression in MDSCs stimulated by HCV RNAcontaining exosomes (33). Reintroduction of miR-124 into peripheral blood mononuclear cells (PBMCs) may reduce the induction of MDSCs by viral nucleic acid-containing exosomes (33).

Activation and differentiation of HSCs into myofibroblasts compose a key mechanism for liver fibrosis. Exosomes released by the $\mathrm{HBx}$-containing cells can stimulate proliferation signals in HSCs (23). miR-192 can be delivered to HSCs through exosomes secreted from HCVreplicating hepatocytes, and upregulate fibroblast markers by upregulating transforming growth factor $\beta 1$ (TGF- $\beta 1$ ) in HSCs (34). Devhare et al. also demonstrated that miR- 19a in HCV-exo was internalized to activate HSC through regulating the suppressor of cytokine signaling (SOCS)signal transducers and activators of transcription 3 (STAT3) axis in vitro (35). Thus, it can be seen that after infection with the hepatitis virus, exosomes and their cargo play a critical role in information communication between hepatocytes and nonparenchymal cells, determining the fate of the virus and the evolution of diseases. Researchers have speculated that exosomes can serve as a new target of viral hepatitis or further fibrosis intervention through the inhibition of their release and the modification of their cargo. Grünvogel et al. (36) demonstrated in vitro that exosomes could transfer the intermediate products of HCV replication, and thus blocking exosomal release might inhibit the replication of HCV through activating toll-like receptor 3 (TLR-3). Moreover, exosomes containing HBV-miR-3 derived from $\mathrm{HBV}$-infected hepatocytes may restrain macrophages from expressing SOCS5 and facilitate M1 polarization by activating the Janus kinase (JAK)/STAT pathway. HBV-miR-3 is capable of enhancing the expression of epidermal growth factor receptor (EGFR) via suppressing SOCS5-mediated ubiquitination, and then stimulating IL-6 secretion to inhibit HBV replication (37). Taken together, the above findings point to the potential value of exosomes in interfering or reversing disease progression after viral infection. However, numerous animal experiments and preclinical studies are still needed before this potential can be confirmed and realized.

\section{Alcoholic liver disease (ALD)}

ALD, which manifests as a wide range of diseases, including alcoholic hepatitis (AH) and cirrhosis, accounts for about $5 \%$ of the global disease burden and $6 \%$ of total global deaths annually. $\mathrm{AH}$ is a syndrome characterized by inflammatory cell infiltration and hepatocyte damage which can stimulate HSCs to secrete excessive extracellular matrix (ECM), such as collagen, and facilitate liver cirrhosis (38) after longterm excessive drinking. With the development of exosome isolation and detection technology, scientists have started to pay attention to their roles in the pathological progression of ALD. A recent study showed that exosome quantity in the serum of healthy people increased after alcohol abuse or long-term drinking (39), with statistically significant correlation with alanine aminotransferase (ALT) levels (40). Ethanol treatment has been found to increase the release of hepatocyte exosomes, which were further preferentially localized in hepatocytes or HSCs, and stimulated the expression of the mRNA involved in exosome biogenesis 
through ceramide or endosomal sorting complexes required for transport (ESCRT) pathways (11). Hence, interference of RNA involved in ESCRT or ceramide pathways in ethanoltreated hepatocytes may reduce exosome production. Meanwhile, in vivo, it has been shown that the exosome inhibitor, GW4869, may suppress intercellular transport of RNA from hepatocytes to target cells (11).

MicroRNAs are the most studied small RNAs in exosomes from mice models and patients with $\mathrm{AH}$. It was demonstrated that miR-30a, miR-30b, miR-122, miR-130a, miR-192, miR744 , and miR-1246 were upregulated in serum exosomes from chronic alcohol-fed mice (40). Babuta et al. (41) further discovered that the inhibition of autophagy and impaired autophagosome and lysosome function were correlated with increased exosome production through the alcohol-related increase of miR-155 in ALD mice models and human livers with ALD. Thus, it may be possible to reduce alcoholinduced liver damage by decreasing the production of exosomes via miR-155 regulation. Transferring exosome miR122 may induce the sensitization of lipopolysaccharide (LPS) and inhibit the heme oxygenase 1 (HO-1) pathway which can suppress the cell injury induced by cytokines and reactive oxygen species. Hence, these effects can be prevented by the exosome-mediated transmission of miR-122 inhibitor (39). Primary HSCs exhibit profibrotic markers, pri-miR-17-92, and connective tissue growth factor (CCN2) (42), and reduce the expression of miR-19b (43) when exposed to alcohol. During HSC activation, the overexpression of exosome miR-19b may change the responsiveness and epigenetic regulatory factors of TGF- $\beta$, further suppressing collagen production (43) (Figure 1, C). Exosomal transfer of miR-214 into the recipient HSCs may also revert the HSC phenotype by directly inhibiting the transcription of CCN2 (42). Understanding these phenomena may be critical to the future development of exosomes as ALD therapeutics.

Other types of cargo in exosomes can also influence the pathological progress of ALD. It was reported that endoplasmic reticulum (ER) stress and oxidative stress may increase the amount of cytochrome P450-2E1 (CYP2E1) in EVs which may promote cell death by stimulating the apoptosis signaling pathway (44). Similarly, alcohol was found to significantly induce CYP2E1 levels of plasma exosomes in an overdrinking mouse model, and these CYP2E1enriched exosomes worsened alcohol-induced hepatotoxicity and monocyte toxicity, which might be reduced by selective CYP2E1 enzyme activity inhibitors (45). In summary, exosomes have the capacity to regulate inflammatory and fibrotic pathways by delivering cargo to target cells in the occurrence and progression of ALD. The above experiments $(44,45)$ provide clues for future clinical treatment, but determining the exact method by which exosomes and their cargo can be used to intervene in the pathogenesis of ALD requires more experimental research.

\section{Nonalcoholic fatty liver diseases (NAFLD)}

NAFLD is a complex disease, ranging from simple steatosis, nonalcoholic steatohepatitis (NASH), cirrhosis, and even to HCC. Its emergence is attributed to systemic inflammation, insulin resistance, and hepatocyte apoptosis. NAFLD is becoming one of the most common chronic liver disorders, with a worldwide prevalence of $25.2 \%$ (46) and an Asian prevalence of $29.6 \%$ (47). Furthermore, the fibrosis progression proportion and HCC incidence were found to be $40.76 \%$ and 0.44 per 1,000 person-years, respectively (46), while the risk ratios of liver-specific and overall mortality of NAFLD were reported to be 1.94 and 1.05 , respectively (46).

Lipotoxicity plays a crucial role in the pathogenesis of NASH through macrophage-associated inflammatory responses, activation of proapoptotic signaling, and angiogenesis $(48,49)$. It may induce lysosomal dysfunction in hepatocytes, then further increase exosome release, causing $M 1$ polarization and macrophage-induced inflammation in an miR-122-5p-dependent (50) or miR192-5p-dependent manner (51). Moreover, palmitic acid (PA) treated (52) or cholesterol-induced hepatocytes (50) were found to display a significant increase in exosome production, showing distinctive miRNA expression patterns. Thus, reducing lipid deposition and inhibiting the release of exosomes may ameliorate the liver inflammation caused by lipotoxicity. Meanwhile, hepatocytes stimulated by lipids were also demonstrated to release EVs containing TNFrelated apoptosis-inducing ligand (TRAIL) (53) and C-X-C motif ligand 10 (CXCL10) (54), which induced macrophage chemotaxis and inflammation phenotype activation. These two effects might be blocked by CXCL10-neutralizing antisera, mixed lineage kinase 3 (MLK3) inhibitor (54), or rho-associated, coiled-coil-containing protein kinase 1 (ROCK1) inhibitor, fasudil (53).

NASH is characterized by neutrophil infiltration around lipotoxic hepatocytes, which is thought to result in the liver inflammation and injury (55). However, recent research (55) has indicated that inflammation and fibrosis could be ameliorated by the communication between neutrophils and hepatocytes through low-density lipoprotein receptor (LDLR)-dependent miR-223-enriched EV transfer. 
Table 1 The roles of exosomal small RNA in HCC therapy

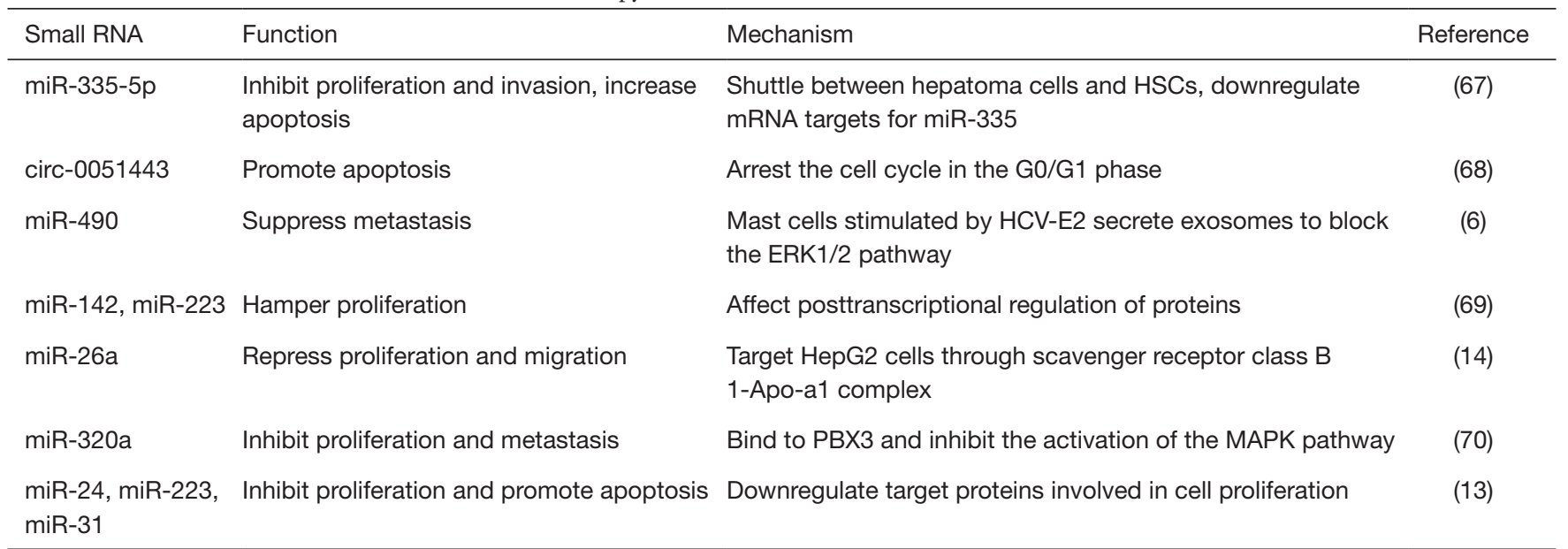

HCC, hepatocellular carcinoma; HSC, hepatic stellate cell; HCV-E2, hepatitis C virus E2 envelope glycoprotein; ERK1/2, extracellular signal-regulated kinase 1/2; PBX3, pre-B-cell leukemia homeobox 3; MAPK, mitogen-activated protein kinase.

Furthermore, Watanabe et al. (56) confirmed that small extracellular vesicles (sEVs) derived from adipose tissuederived MSCs (AD-MSCs) could attenuate inflammation and inhibit liver fibrosis in a rapid NASH fibrosis model (Figure 1, C). Consequently, exosomes and their cargo are significant to the pathogenesis of NAFLD, but may also be attractive therapeutic tools for treating liver diseases. In the future, making full use of exosomes may provide scientists with novel strategies for the treatment of nonalcoholic liver disease.

\section{Hepatocellular carcinoma}

HCC, the most common type of liver cancer, is a major global public health issue of concern. Chronic HBV and $\mathrm{HCV}$ infections are generally considered to be risk factors for HCC, and account for $56 \%$ and $20 \%$ of cases, respectively $(57,58)$. Only a small portion of HCC patients who have underlying basic chronic liver diseases and cirrhosis may be rescued by resection or liver transplantation (59) due to insufficient donors, financial considerations, and other factors. The survival of advanced HCC still remains poor because of insensitivity or drug resistance to chemotherapy (60). Consequently, it is necessary to explore a new treatment for HCC. Exosomes are extremely useful for the horizontal delivery of multiple RNAs and protein molecules to adjacent or distant cells via paracrine and autocrine forms, and are implicated in the mechanisms of the occurrence and progression of HCC tumor, including angiogenesis, epithelial-mesenchymal transition (EMT), immune escape, and chemotherapy drug resistance $(61,62)$. He et al. (61) conducted proteomic analysis and RNA sequencing of the cargo in HCC-derived and immortalized hepatocyte-derived exosomes. The results showed exosomes carried many protumorigenic proteins and RNAs, such as caveolins, RRAS, CLND3e, and S100A4. Exosome-enriched pathogenic genes derived from hepatoma cells could significantly enhance the migration and invasion of normal hepatocytes by triggering mitogen-activated protein kinase (MAPK) and phosphatidylinositol 3 kinase (PI3K)/protein kinase B (AKT) signaling pathways, as well as increasing secretion of active matrix metalloproteinases-2 (MMP-2) and matrix metalloproteinases-9 (MMP-9) (61). Exosomes may exert hepatoma inhibitory effects by the overexpression of a key regulator of exosome biogenesis-vacuolar protein sorting 4 homolog A (Vps4A) to inactivate PI3K/AKT pathway and utilize exosomes to modulate the secretion and uptake of miRNAs (63). Hepatoma cell-secreted exosomal miR-210 (64) and miR-155 (65) may promote tumor angiogenesis of endothelial cells and elevate the proliferation of HCC cells. Conversely, repression or knockdown of these two miRNAs may have the opposite effect, inhibiting angiogenic activity in $\mathrm{HCC}(64,65)$. Furthermore, knockdown of phosphatase and tensin homolog deleted on chromosome 10 (PTEN) may attenuate the proliferation of HCC cells treated with the exosomal miR-155 (66). Earlier studies (7) showed that exosomes could transfer RNA between cells, acting as a good adjuster to diseases (Table 1). 
Wang et al. (67) demonstrated in vivo and in vitro that miR$335-5 \mathrm{p}$ could be delivered by exosomes to hepatoma cells, so as to inhibit their proliferation and invasion and increase apoptosis (Figure 1, D). Meanwhile, Chen et al. (68) showed that when exosomal circular RNA (circRNA)-0051443 was transmitted to hepatoma cells, the cell cycle ceased.

Moreover, immunocyte-derived exosomes can exert a great influence on antitumor immune responses (6). For example, mast cells stimulated by HCV-E2 envelope glycoprotein (HCV-E2) are capable of secreting numerous exosomes rich in miR-490, which can be transferred into hepatoma cells and suppress their metastasis through blocking the extracellular signal-regulated kinase 1/2 (ERK1/2) pathway (6). MiR-142 and miR-223 expressed in macrophages can be transferred by exosomes to affect posttranscriptional regulation of proteins in HCC cells which hampers the proliferation of these cancer cells (69). Dendritic cells (DCs) play a key role in both innate and adaptive immune responses, and their maturation and activation can be disturbed by the HCC-induced tumor microenvironment (71). Tumor-derived exosomes can activate DCs, stimulate the proliferation of immature $\mathrm{T}$ cells, and induce $\mathrm{T}$ cells to differentiate into antigenspecific cytotoxic $\mathrm{T}$ lymphocytes (CTLs), thus increasing antineoplastic efficacy $(72,73)$ (Figure 1, D). Rao et al. reported that the tumor immune microenvironment in HCC mice was significantly improved upon application of HCCderived exosome-pulsed DCs. The number of T cells and the level of interferon gamma (IFN- $\gamma$ ) increased, while IL10 and TGF- $\beta$ decreased, which eventually resulted in tumor growth inhibition and a strong immune response (74). They also found that the exosomes released by alpha-fetoprotein (AFP)-expressing DCs had antitumor properties similar to those of HCC-derived exosome-pulsed DCs, thus providing a cell-free vehicle for tumor immunotherapy (75).

Accumulating evidence has shown that cancer cells can release exosomes to promote carcinogenesis and the resistance or insensitivity to multiple chemotherapeutic drugs, and thus a greater research focus has been placed on exosome-based drug delivery to inhibit cancer development through interference techniques (76). For instance, some studies have found that the exposure of HCC cells to sorafenib increased long intergenic noncoding RNAVLDLR (linc-VLDLR) and long noncoding RNA ROR (lncRNA ROR) expression in cells and EVs. RNA interference-mediated knockdown of these two lncRNAs increased chemotherapy-induced cytotoxicity and apoptosis, leading to enhanced chemosensitivity on $\operatorname{HCC}(77,78)$. Some preclinical findings have suggested that exosome- mediated drug delivery has good prospects in cancer treatment. For example, engineered exosomes packed with miR-26a were shown to selectively target HepG2 cells through scavenger receptor class B 1-Apo-a1 complex, so as to repress cell proliferation and migration (14). Furthermore, when miR-320a-enriched exosomes were injected into rats via the caudal vein, it was observed that the proliferation and metastasis of hepatoma cells were effectively inhibited via binding to the downstream target pre-B-cell leukemia homeobox 3 (PBX3) (70), further inhibiting the activation of the MAPK pathway (70). MiR24, miR-223, and miR-31 delivered by exosomes can also restrain the growth and invasion of HCC and increase apoptosis, exerting potential antitumor activity in vivo (13).

These preclinical experiments have clarified the functions of exosomes and their cargo in the inhibition of HCC progression. Due to the advantages of low immunogenicity and toxicity, and the natural release or uptake by tumor cells $(76,79)$, exosomes are expected to become an attractive therapeutic strategy for the treatment of HCC in the future.

\section{MSC-derived exosomes}

MSCs are multipotent stromal cells possessing various biological functions, such as self-renewal, multilineage differentiation, and anti-inflammation $(80,81)$. Recently, use of MSC-derived exosomes functioning as potent therapeutic vehicles has become a promising strategy for various diseases $(80,81)$ (Table 2). Jiang et al. found that human umbilical cord MSC (UC-MSC)-derived exosomes might alleviate acute liver injury and fibrosis induced by carbon tetrachloride $\left(\mathrm{CCl}_{4}\right)$ in mouse models via antioxidant potentials (82). MiR-455-3p-containing exosomes released by UC-MSCs stimulated with IL-6 could suppress macrophage activation and reduce cytokine production by targeting PI3K signaling in a chemical liver injury animal model, and consequently ameliorate liver histology and retrieve function (83) (Figure 1, E). Furthermore, Rong et al. applied other exosomes derived from human bone marrow MSCs (BM-MSCs) in the treatment of liver fibrosis induced by $\mathrm{CCl}_{4}$. The results showed fibrosis amelioration and HSC activation was inhibited via Wnt/ $\beta$-catenin pathway (85) (Figure 1, E). BM-MSC-derived exosomes may also attenuate the hepatic inflammatory response and reduce the release of inflammatory cytokines from macrophages in autoimmune hepatitis, which may be associated with the expression levels of miR-223-3p and STAT3 in macrophages (89). 
Table 2 The therapeutic effect of MSC-derived exosomes in liver diseases

\begin{tabular}{llll}
\hline Origin & Disease & Function & \\
\hline UC-MSCs & Acute liver injury and fibrosis & Antioxidant potentials & Reference \\
UC-MSCs & Chemical liver injury & Suppress macrophage activation and reduce cytokine production & (82) \\
UC-MSCs & ALF & Inhibit NLRP3 activation in macrophage and decrease proinflammatory cytokines & (84) \\
& & level & \\
BM-MSCs & Liver fibrosis induced by CCl & Inhibit HSC activation via Wnt/ $\beta$-Catenin pathway \\
BM-MSCs & ALF & Decrease the levels of cleaved caspase 3 and Bax, upregulate the expression of & (86) \\
& & Bcl-2 & \\
BM-MSCs & I/R injury & Inhibit Th17 cells and induce Treg cells \\
AD-MSCs & HCC & Improve the sensitivity of chemotherapeutic drugs & (87) \\
\hline
\end{tabular}

MSC, mesenchymal stem cell; UC-MSC, umbilical cord mesenchymal stem cell; BM-MSC, bone marrow mesenchymal stem cell; AD-MSC, adipose-derived mesenchymal stem cell; ALF, acute liver failure; NLRP3, nucleotide-binding and oligomerization domain-like receptor 3; $\mathrm{CCl}_{4}$, carbon tetrachloride; I/R injury, ischemia/reperfusion injury; HCC, hepatocellular carcinoma; HSC, hepatic stellate cell.

Acute liver failure (ALF) is a fatal illness with high mortality, which can only be controlled by liver transplantation and artificial liver therapy (90). However, various constraints including ischemia/reperfusion (I/R) injury have prompted researchers to explore alternative treatments. MSC exosomes have shown the potential to prolong or save lives. Studies have demonstrated that TNF-alpha pretreatment of UC-MSC-derived exosomes (T-Exo) and adipose tissue-derived MSC exosomes (AMSC-exo) decreased serum ALT, aspartate transaminase (AST), and proinflammatory cytokine level, inhibited activation of nucleotide-binding and oligomerization domain-like receptor 3 (NLRP3) in macrophages, and reduced pathological liver damage caused by ALF $(84,91)$. Furthermore, anti-inflammatory-related miR-299-3p packaged into exosomes is upregulated by TNF-alphastimulated MSCs, which exerts a therapeutic effect (84); meanwhile, miR-17 is elevated in AMSC-exo through suppressing thioredoxin-interacting protein/nucleotidebinding and oligomerization domain-like receptor protein 3 (TXNIP/NLRP3) signaling pathway (91). In addition to having anti-inflammation effects, BM-MSC exosomes applied in ALF may also significantly decrease the levels of cleaved caspase- 3 and Bax, and upregulate the expression of Bcl-2, which can attenuate hepatocyte apoptosis and promote autophagy (86).

$\mathrm{I} / \mathrm{R}$ injury, including inflammation, necrosis, and apoptosis, is the main problem in liver transplantation (92). MiR-20a secreted by MSC exosomes can bind to the 3' untranslated region (3' UTR) of Fas and Beclin-I to regulate the gene expression involving apoptosis and autophagy (93). Furthermore, BM-MSC-differentiated hepatocyte-like cell exosomes (MSC-Heps-exo) may alleviate hepatic I/R injury effectively and reduce hepatocyte apoptosis and the levels of liver enzyme in vivo and in vitro (92). Lai et al. (87) found BM-MSC-exo could also inhibit Th17 cells and induce regulatory $\mathrm{T}$ cells (Tregs) to reduce injury and ameliorate the survival of chronic graft-versus-host disease (cGVHD). In addition, studies (94) have demonstrated that fetal liver MSC-derived exosomes suppress proliferation, activation, and cytotoxicity of NK cells via TGF- $\beta /$ Smad $2 / 3$ signaling in allogeneic reactions, and that applying anti-TGF- $\beta$ antibody may restore NK cell function. Therefore, using MSC-derived exosomes to ameliorate I/R injury shows considerable promise.

Currently, the application of MSCs exosomes in liver cancer has become a key goal of clinical research, and a large number of related studies have been conducted. Jiang et al. (82) administered UC-MSC-derived exosomes into $\mathrm{CCl}_{4}$-induced liver tumor in vivo. They found these exosomes could provide more antioxidant effects than bifendate treatment and exerted hepatoprotective effects, subsequently restraining the growth of tumors (82). Exosomes from adipose-derived mesenchymal stem cells (AD-MSCs) could promote NK cells to exert antitumor roles on rat HCC, thereby facilitating low-grade tumor differentiation and inhibiting tumor growth (95) (Figure 1, E). Accumulating evidence has indicated that miR-122 has the property of promoting the chemosensitivity of HCC cells. Hence, Lou et al. chose AD-MSC exosomes as biological 
vehicles for miR-122 delivery. The results showed that miR-122-transfected AMSCs successfully mediated miR122 transmission between AMSCs and HCC cells, thereby inducing tumor cells to be sensitive to chemotherapeutic drugs (88). Meanwhile, injecting MSC-derived exosomes containing miR-122 into tumor could effectively enhance the antitumor efficacy of sorafenib (88) (Figure 1, E). Collectively, MSC-exos are being increasingly considered as attractive candidates to control disease progression and improve liver function.

\section{Conclusions}

With more evidence being generated from preclinical and animal experiments, researchers have begun to draw the blueprint for the clinical application of exosomes and their cargo in the treatment of liver diseases. Although the above results are encouraging, there are still many problems that remain to be solved. The establishment of standard methods to isolate and identify the types and sources of valuable exosomes is the first step. The quality and sufficient quantity of these exosomes need to be ensured in order to optimize the therapeutic functions of exosomes during treatment. Secondly, the mechanisms by which exosomes are released by cells and recognized and fused by target cells need to be fully understood. Thirdly, comprehensive examination of the genes and protein functions carried by exosomes are needed, as some molecules demonstrate therapeutic effects, while others do not, or may even be deleterious. Once the properties of these molecules are grasped, valuable traits may be exploited, while others can be discarded. Research on exosomes have opened a door to discovering the mechanisms underlying the occurrence and development of various liver diseases, and providing a new drug vehicle with low immunogenicity and high biocompatibility. There is still a long way to go before clinical application can be actualized, and more experimental evidence is needed to support future clinical research.

\section{Acknowledgments}

The authors thank Lan Guo and Panzhi Wang for English language editing.

Funding: This work was supported by funding from the National Science and Technology Major Project (No. 2017ZX10203201) and the opening foundation of the State Key Laboratory for Diagnosis and Treatment of Infectious Diseases and Collaborative Innovation Center for Diagnosis and Treatment of Infectious Diseases, First Affiliated Hospital, Zhejiang University School of Medicine (No. 2015KF04).

\section{Footnote}

Provenance and Peer Review: This article was commissioned by the Guest Editors (Ralf Weiskirchen and Wolfgang Stremmel) for the series "Unresolved Basic Issues in Hepatology" published in Annals of Translational Medicine. The article has undergone external peer review.

Conflicts of Interest: All authors have completed the ICMJE uniform disclosure form (available at http://dx.doi. org/10.21037/atm-20-5422). The series "Unresolved Basic Issues in Hepatology" was commissioned by the editorial office without any funding or sponsorship. The authors have no other conflicts of interest to declare.

Ethical Statement: The authors are accountable for all aspects of the work in ensuring that questions related to the accuracy or integrity of any part of the work are appropriately investigated and resolved.

Open Access Statement: This is an Open Access article distributed in accordance with the Creative Commons Attribution-NonCommercial-NoDerivs 4.0 International License (CC BY-NC-ND 4.0), which permits the noncommercial replication and distribution of the article with the strict proviso that no changes or edits are made and the original work is properly cited (including links to both the formal publication through the relevant DOI and the license). See: https://creativecommons.org/licenses/by-nc-nd/4.0/.

\section{References}

1. Pan BT, Teng $\mathrm{K}, \mathrm{Wu} \mathrm{C}$, et al. Electron microscopic evidence for externalization of the transferrin receptor in vesicular form in sheep reticulocytes. J Cell Biol 1985;101:942-8.

2. Zhang H, Deng T, Ge S, et al. Exosome circRNA secreted from adipocytes promotes the growth of hepatocellular carcinoma by targeting deubiquitination-related USP7. Oncogene 2019;38:2844-59.

3. Kalluri R, LeBleu VS. The biology, function, and biomedical applications of exosomes. Science 2020;367:eaau6977.

4. Théry C, Zitvogel L, Amigorena S. Exosomes: 
composition, biogenesis and function. Nat Rev Immunol 2002;2:569-79.

5. David G, Zimmermann P. Heparanase Involvement in Exosome Formation. Adv Exp Med Biol 2020; 1221:285-307.

6. Wu Q, Zhou L, Lv D, et al. Exosome-mediated communication in the tumor microenvironment contributes to hepatocellular carcinoma development and progression. J Hematol Oncol 2019;12:53.

7. Raposo G, Stoorvogel W. Extracellular vesicles: exosomes, microvesicles, and friends. J Cell Biol 2013;200:373-83.

8. Liu H, Li B. The functional role of exosome in hepatocellular carcinoma. J Cancer Res Clin Oncol 2018;144:2085-95.

9. Pegtel DM, Gould SJ. Exosomes. Annu Rev Biochem 2019;88:487-514.

10. Nojima H, Freeman CM, Schuster RM, et al. Hepatocyte exosomes mediate liver repair and regeneration via sphingosine-1-phosphate. J Hepatol 2016;64:60-8.

11. Chen L, Chen R, Kemper S, et al. Pathways of production and delivery of hepatocyte exosomes. J Cell Commun Signal 2018;12:343-57.

12. Sung S, Kim J, Jung Y. Liver-Derived Exosomes and Their Implications in Liver Pathobiology. Int J Mol Sci 2018;19:3715.

13. Fonsato V, Collino F, Herrera MB, et al. Human liver stem cell-derived microvesicles inhibit hepatoma growth in SCID mice by delivering antitumor microRNAs. Stem Cells 2012;30:1985-98.

14. Liang G, Kan S, Zhu Y, et al. Engineered exosomemediated delivery of functionally active miR-26a and its enhanced suppression effect in HepG2 cells. Int J Nanomedicine 2018;13:585-99.

15. Borrelli DA, Yankson K, Shukla N, et al. Extracellular vesicle therapeutics for liver disease. J Control Release 2018;273:86-98.

16. Balaphas A, Meyer J, Sadoul R, et al. Extracellular vesicles: Future diagnostic and therapeutic tools for liver disease and regeneration. Liver Int 2019;39:1801-17.

17. He X, Yu J, Xiong L, et al. Exosomes derived from liver cancer cells reprogram biological behaviors of $\mathrm{LO} 2$ cells by transferring Linc-ROR. Gene 2019;719:144044.

18. Villa F, Quarto R, Tasso R. Extracellular Vesicles as Natural, Safe and Efficient Drug Delivery Systems. Pharmaceutics 2019;11:557.

19. Deng L, Gan X, Ito M, et al. Peroxiredoxin 1, a Novel HBx-Interacting Protein, Interacts with Exosome Component 5 and Negatively Regulates Hepatitis B Virus
(HBV) Propagation through Degradation of HBV RNA. J Virol 2019;93:e02203-18.

20. Mohd-Ismail NK, Lim Z, Gunaratne J, et al. Mapping the Interactions of HBV cccDNA with Host Factors. Int J Mol Sci 2019;20:4276.

21. Guo H, Jiang D, Zhou T, et al. Characterization of the intracellular deproteinized relaxed circular DNA of hepatitis B virus: an intermediate of covalently closed circular DNA formation. J Virol 2007;81:12472-84.

22. Allweiss L, Dandri M. The Role of cccDNA in HBV Maintenance. Viruses 2017;9:156.

23. Kapoor NR, Chadha R, Kumar S, et al. The HBx gene of hepatitis $\mathrm{B}$ virus can influence hepatic microenvironment via exosomes by transferring its mRNA and protein. Virus Res 2017;240:166-74.

24. Jia X, Chen J, Megger DA, et al. Label-free Proteomic Analysis of Exosomes Derived from Inducible Hepatitis B Virus-Replicating HepAD38 Cell Line. Mol Cell Proteomics 2017;16:S144-60.

25. Ramakrishnaiah V, Thumann C, Fofana I, et al. Exosomemediated transmission of hepatitis $\mathrm{C}$ virus between human hepatoma Huh7.5 cells. Proc Natl Acad Sci U S A 2013;110:13109-13.

26. Yang $Y$, Han Q, Hou Z, et al. Exosomes mediate hepatitis $B$ virus (HBV) transmission and NK-cell dysfunction. Cell Mol Immunol 2017;14:465-75.

27. Wang J, Chen R, Lu FM. Exosome-mediated CRISPR/ Cas9 system targets to cut the intercellular transmission function of hepatitis B virus genome. Zhonghua Gan Zang Bing Za Zhi 2019;27:610-4.

28. Shen J, Huang $\mathrm{CK}, \mathrm{Yu} \mathrm{H}$, et al. The role of exosomes in hepatitis, liver cirrhosis and hepatocellular carcinoma. J Cell Mol Med 2017;21:986-92.

29. Kouwaki T, Fukushima Y, Daito T, et al. Extracellular Vesicles Including Exosomes Regulate Innate Immune Responses to Hepatitis B Virus Infection. Front Immunol 2016;7:335.

30. Chen TC, Hsieh CH, Sarnow P. Supporting Role for GTPase Rab27a in Hepatitis C Virus RNA Replication through a Novel miR-122-Mediated Effect. PLoS Pathog 2015;11:e1005116.

31. Elgner F, Ren H, Medvedev R, et al. The Intracellular Cholesterol Transport Inhibitor U18666A Inhibits the Exosome-Dependent Release of Mature Hepatitis C Virus. J Virol 2016;90:11181-96.

32. Cosset FL, Dreux M. HCV transmission by hepatic exosomes establishes a productive infection. J Hepatol 2014;60:674-5. 
33. Wang L, Cao D, Wang L, et al. HCV-associated exosomes promote myeloid-derived suppressor cell expansion via inhibiting miR-124 to regulate $T$ follicular cell differentiation and function. Cell Discov 2018;4:51.

34. Kim JH, Lee CH, Lee SW. Exosomal Transmission of MicroRNA from HCV Replicating Cells Stimulates Transdifferentiation in Hepatic Stellate Cells. Mol Ther Nucleic Acids 2019;14:483-97.

35. Devhare PB, Sasaki R, Shrivastava S, et al. ExosomeMediated Intercellular Communication between Hepatitis C Virus-Infected Hepatocytes and Hepatic Stellate Cells. J Virol 2017;91:e02225-16. Erratum in: J Virol. 2017 Apr 28;91(10):e00349-17. doi: 10.1128/JVI.00349-17. Print 2017 May 15.

36. Grünvogel O, Colasanti O, Lee JY, et al. Secretion of Hepatitis C Virus Replication Intermediates Reduces Activation of Toll-Like Receptor 3 in Hepatocytes. Gastroenterology 2018;154:2237-2251.e16..

37. Zhao X, Sun L, Mu T, et al. An HBV-encoded miRNA activates innate immunity to restrict $\mathrm{HBV}$ replication. J Mol Cell Biol 2020;12:263-76.

38. Friedman SL. Mechanisms of hepatic fibrogenesis. Gastroenterology 2008;134:1655-69.

39. Momen-Heravi F, Bala S, Kodys K, et al. Exosomes derived from alcohol-treated hepatocytes horizontally transfer liver specific miRNA-122 and sensitize monocytes to LPS. Sci Rep 2015;5:9991.

40. Momen-Heravi F, Saha B, Kodys K, et al. Increased number of circulating exosomes and their microRNA cargos are potential novel biomarkers in alcoholic hepatitis. J Transl Med 2015;13:261.

41. Babuta M, Furi I, Bala S, et al. Dysregulated Autophagy and Lysosome Function Are Linked to Exosome Production by Micro-RNA 155 in Alcoholic Liver Disease. Hepatology 2019;70:2123-41.

42. Chen L, Charrier A, Zhou Y, et al. Epigenetic regulation of connective tissue growth factor by MicroRNA-214 delivery in exosomes from mouse or human hepatic stellate cells. Hepatology 2014;59:1118-29.

43. Brandon-Warner E, Feilen NA, Culberson CR, et al. Processing of miR17-92 Cluster in Hepatic Stellate Cells Promotes Hepatic Fibrogenesis During Alcohol-Induced Injury. Alcohol Clin Exp Res 2016;40:1430-42.

44. Cho YE, Mezey E, Hardwick JP, et al. Increased ethanolinducible cytochrome P450-2E1 and cytochrome $\mathrm{P} 450$ isoforms in exosomes of alcohol-exposed rodents and patients with alcoholism through oxidative and endoplasmic reticulum stress. Hepatol Commun
2017;1:675-90.

45. Rahman MA, Kodidela S, Sinha N, et al. Plasma exosomes exacerbate alcohol- and acetaminophen-induced toxicity via CYP2E1 pathway. Sci Rep 2019;9:6571.

46. Younossi ZM, Koenig AB, Abdelatif D, et al. Global epidemiology of nonalcoholic fatty liver disease-Metaanalytic assessment of prevalence, incidence, and outcomes. Hepatology 2016;64:73-84.

47. Li J, Zou B, Yeo YH, et al. Prevalence, incidence, and outcome of non-alcoholic fatty liver disease in Asia, 19992019: a systematic review and meta-analysis. Lancet Gastroenterol Hepatol 2019;4:389-98.

48. Hirsova P, Ibrahim SH, Gores GJ, et al. Lipotoxic lethal and sublethal stress signaling in hepatocytes: relevance to NASH pathogenesis. J Lipid Res 2016;57:1758-70. Erratum in: J Lipid Res. 2017 Jan;58(1):299. doi: 10.1194/ jlr.R066357ERR.

49. Povero D, Eguchi A, Niesman IR, et al. Lipid-induced toxicity stimulates hepatocytes to release angiogenic microparticles that require Vanin-1 for uptake by endothelial cells. Sci Signal 2013;6:ra88.

50. Zhao Z, Zhong L, Li P, et al. Cholesterol impairs hepatocyte lysosomal function causing M1 polarization of macrophages via exosomal miR-122-5p. Exp Cell Res 2020;387:111738.

51. Liu XL, Pan Q, Cao HX, et al. Lipotoxic HepatocyteDerived Exosomal MicroRNA 192-5p Activates Macrophages Through Rictor/Akt/Forkhead Box Transcription Factor O1 Signaling in Nonalcoholic Fatty Liver Disease. Hepatology 2020;72:454-69.

52. Lee YS, Kim SY, Ko E, et al. Exosomes derived from palmitic acid-treated hepatocytes induce fibrotic activation of hepatic stellate cells. Sci Rep 2017;7:3710.

53. Hirsova P, Ibrahim SH, Krishnan A, et al. Lipid-Induced Signaling Causes Release of Inflammatory Extracellular Vesicles From Hepatocytes. Gastroenterology 2016;150:956-67.

54. Ibrahim $\mathrm{SH}$, Hirsova $\mathrm{P}$, Tomita $\mathrm{K}$, et al. Mixed lineage kinase 3 mediates release of $\mathrm{C}-\mathrm{X}-\mathrm{C}$ motif ligand 10-bearing chemotactic extracellular vesicles from lipotoxic hepatocytes. Hepatology 2016;63:731-44.

55. He Y, Rodrigues RM, Wang X, et al. Neutrophilto-hepatocyte communication via LDLR-dependent miR-223-enriched extracellular vesicle transfer ameliorates nonalcoholic steatohepatitis. J Clin Invest 2021;131:e141513.

56. Watanabe T, Tsuchiya A, Takeuchi S, et al. Development of a non-alcoholic steatohepatitis model with rapid 
accumulation of fibrosis, and its treatment using mesenchymal stem cells and their small extracellular vesicles. Regen Ther 2020;14:252-61.

57. Pezzuto F, Buonaguro L, Buonaguro FM, et al. The Role of Circulating Free DNA and MicroRNA in Non-Invasive Diagnosis of HBV- and HCV-Related Hepatocellular Carcinoma. Int J Mol Sci 2018;19:1007.

58. Maucort-Boulch D, de Martel C, Franceschi S, et al. Fraction and incidence of liver cancer attributable to hepatitis $\mathrm{B}$ and $\mathrm{C}$ viruses worldwide. Int J Cancer 2018;142:2471-7.

59. Xu X, Chen J, Wei Q, et al. Clinical practice guidelines on liver transplantation for hepatocellular carcinoma in China (2018 edition). Hepatobiliary Pancreat Dis Int 2019;18:307-12.

60. Li Z, Zhu JY. Hepatocellular carcinoma: Current situation and challenge. Hepatobiliary Pancreat Dis Int 2019;18:303-4.

61. He M, Qin H, Poon TC, et al. Hepatocellular carcinomaderived exosomes promote motility of immortalized hepatocyte through transfer of oncogenic proteins and RNAs. Carcinogenesis 2015;36:1008-18.

62. Abudoureyimu M, Zhou H, Zhi Y, et al. Recent progress in the emerging role of exosome in hepatocellular carcinoma. Cell Prolif 2019;52:e12541.

63. Wei JX, Lv LH, Wan YL, et al. Vps4A functions as a tumor suppressor by regulating the secretion and uptake of exosomal microRNAs in human hepatoma cells. Hepatology 2015;61:1284-94.

64. Lin XJ, Fang JH, Yang XJ, et al. Hepatocellular Carcinoma Cell-Secreted Exosomal MicroRNA-210 Promotes Angiogenesis In Vitro and In Vivo. Mol Ther Nucleic Acids 2018;11:243-52.

65. Matsuura Y, Wada H, Eguchi H, et al. Exosomal miR155 Derived from Hepatocellular Carcinoma Cells Under Hypoxia Promotes Angiogenesis in Endothelial Cells. Dig Dis Sci 2019;64:792-802.

66. Sun JF, Zhang D, Gao CJ, et al. Exosome-Mediated MiR155 Transfer Contributes to Hepatocellular Carcinoma Cell Proliferation by Targeting PTEN. Med Sci Monit Basic Res 2019;25:218-28.

67. Wang F, Li L, Piontek K, et al. Exosome miR-335 as a novel therapeutic strategy in hepatocellular carcinoma. Hepatology 2018;67:940-54.

68. Chen W, Quan Y, Fan S, et al. Exosome-transmitted circular RNA hsa_circ_0051443 suppresses hepatocellular carcinoma progression. Cancer Lett 2020;475:119-28.

69. Aucher A, Rudnicka D, Davis DM. MicroRNAs transfer from human macrophages to hepato-carcinoma cells and inhibit proliferation. J Immunol 2013;191:6250-60.

70. Zhang Z, Li X, Sun W, et al. Loss of exosomal miR-320a from cancer-associated fibroblasts contributes to HCC proliferation and metastasis. Cancer Lett 2017;397:33-42.

71. Motta JM, Rumjanek VM. Sensitivity of Dendritic Cells to Microenvironment Signals. J Immunol Res 2016;2016:4753607.

72. Chen R, Xu X, Tao Y, et al. Exosomes in hepatocellular carcinoma: a new horizon. Cell Commun Signal 2019;17:1.

73. Li J, Huang S, Zhou Z, et al. Exosomes derived from rAAV/AFP-transfected dendritic cells elicit specific $T$ cell-mediated immune responses against hepatocellular carcinoma. Cancer Manag Res 2018;10:4945-57.

74. Rao Q, Zuo B, Lu Z, et al. Tumor-derived exosomes elicit tumor suppression in murine hepatocellular carcinoma models and humans in vitro. Hepatology 2016;64:456-72.

75. Lu Z, Zuo B, Jing R, et al. Dendritic cell-derived exosomes elicit tumor regression in autochthonous hepatocellular carcinoma mouse models. J Hepatol 2017;67:739-48.

76. Tai YL, Chen KC, Hsieh JT, et al. Exosomes in cancer development and clinical applications. Cancer Sci 2018;109:2364-74.

77. Takahashi K, Yan IK, Kogure T, et al. Extracellular vesicle-mediated transfer of long non-coding RNA ROR modulates chemosensitivity in human hepatocellular cancer. FEBS Open Bio 2014;4:458-67.

78. Takahashi K, Yan IK, Wood J, et al. Involvement of extracellular vesicle long noncoding RNA (linc-VLDLR) in tumor cell responses to chemotherapy. Mol Cancer Res 2014;12:1377-87.

79. Kim MS, Haney MJ, Zhao Y, et al. Development of exosome-encapsulated paclitaxel to overcome MDR in cancer cells. Nanomedicine 2016;12:655-64.

80. Xunian Z, Kalluri R. Biology and therapeutic potential of mesenchymal stem cell-derived exosomes. Cancer Sci 2020;111:3100-10.

81. Yu B, Shao H, Su C, et al. Exosomes derived from MSCs ameliorate retinal laser injury partially by inhibition of MCP-1. Sci Rep 2016;6:34562.

82. Jiang W, Tan Y, Cai M, et al. Human Umbilical Cord MSC-Derived Exosomes Suppress the Development of $\mathrm{CCl}(4)-$ Induced Liver Injury through Antioxidant Effect. Stem Cells Int 2018;2018:6079642.

83. Shao M, Xu Q, Wu Z, et al. Exosomes derived from human umbilical cord mesenchymal stem cells ameliorate IL-6-induced acute liver injury through miR-455-3p. Stem Cell Res Ther 2020;11:37. 
84. Zhang S, Jiang L, Hu H, et al. Pretreatment of exosomes derived from hUCMSCs with TNF- $\alpha$ ameliorates acute liver failure by inhibiting the activation of NLRP3 in macrophage. Life Sci 2020;246:117401.

85. Rong X, Liu J, Yao X, et al. Human bone marrow mesenchymal stem cells-derived exosomes alleviate liver fibrosis through the $\mathrm{Wnt} / \beta$-catenin pathway. Stem Cell Res Ther 2019;10:98.

86. Zhao S, Liu Y, Pu Z. Bone marrow mesenchymal stem cell-derived exosomes attenuate D-GaIN/LPS-induced hepatocyte apoptosis by activating autophagy in vitro. Drug Des Devel Ther 2019;13:2887-97.

87. Lai P, Chen X, Guo L, et al. A potent immunomodulatory role of exosomes derived from mesenchymal stromal cells in preventing cGVHD. J Hematol Oncol 2018;11:135.

88. Lou G, Song X, Yang F, et al. Exosomes derived from miR-122-modified adipose tissue-derived MSCs increase chemosensitivity of hepatocellular carcinoma. J Hematol Oncol 2015;8:122.

89. Lu FB, Chen DZ, Chen L, et al. Attenuation of Experimental Autoimmune Hepatitis in Mice with Bone Mesenchymal Stem Cell-Derived Exosomes Carrying MicroRNA-223-3p. Mol Cells 2019;42:906-18.

90. Zhang S, Hou Y, Yang J, et al. Application of mesenchymal stem cell exosomes and their drug-loading systems in acute liver failure. J Cell Mol Med 2020;24:7082-93.

Cite this article as: Ding J, Wang J, Chen J. Exosomes as therapeutic vehicles in liver diseases. Ann Transl Med 2021;9(8):735. doi: 10.21037/atm-20-5422
91. Liu Y, Lou G, Li A, et al. AMSC-derived exosomes alleviate lipopolysaccharide/d-galactosamine-induced acute liver failure by miR-17-mediated reduction of TXNIP/ NLRP3 inflammasome activation in macrophages. EBioMedicine 2018;36:140-50.

92. Yang B, Duan W, Wei L, et al. Bone Marrow Mesenchymal Stem Cell-Derived Hepatocyte-Like Cell Exosomes Reduce Hepatic Ischemia/Reperfusion Injury by Enhancing Autophagy. Stem Cells Dev 2020;29:372-9.

93. Zhang L, Song Y, Chen L, et al. MiR-20a-containing exosomes from umbilical cord mesenchymal stem cells alleviates liver ischemia/reperfusion injury. J Cell Physiol 2020;235:3698-710.

94. Fan Y, Herr F, Vernochet A, et al. Human Fetal Liver Mesenchymal Stem Cell-Derived Exosomes Impair Natural Killer Cell Function. Stem Cells Dev 2019;28:44-55.

95. Ko SF, Yip HK, Zhen YY, et al. Adipose-Derived Mesenchymal Stem Cell Exosomes Suppress Hepatocellular Carcinoma Growth in a Rat Model: Apparent Diffusion Coefficient, Natural Killer T-Cell Responses, and Histopathological Features. Stem Cells Int 2015;2015:853506.

(English Language Editor: J. Gray) 